\title{
Migración en la ciudad: Impacto urbano, escenarios de sostenibilidad
}

Migration in the city: Urban

Impact on Sustainable Scenarios

Migrations urbaines :

Impact et scénario

de durabilité

Migraçãa na cidade:

Impacto urbano cenários

de sustentabilidade

"Docente asociado Núcleo de Competencias Socio Humanísticas, Universidad EAN. Investigador con CVLAC Grupo Políticas públicas y responsabilidad social, 


\section{RESUMEN}

El estudio de los escenarios de planeación urbana en el contexto de la productividad y la competitividad empresarial, para el caso de la ciudad de Bogotá, sirven de soporte para el análisis, la formulación y el direccionamiento de la políticas públicas que permitan involucrar a la población que sufre un desplazamiento interno.

El artículo busca identificar el impacto urbano y la formulación de las políticas públicas migratorias que se aplican como efecto de regulación; es importante tenerlas en cuenta para el buen funcionamiento y desarrollo del mismo, puesto que las personas desplazadas, que están presentes en la ciudad, no siempre contribuyen positivamente al desarrollo urbano y esto hace que la efectividad de las políticas públicas implementadas no siempre se cumplan o se implementen en su cabalidad. Esto invita a que se formulen e identifiquen las políticas públicas para establecer un control de las personas que tienen el derecho de habitar.

Puede que muchas de estas personas no quieran influir negativamente y pasar desapercibidas, sin embargo, es importante y necesario reconocerlas e identificarlas para mantener un orden en la sociedad.

La ciudad, en su desarrollo, busca la planeación, se adapta y evoluciona con el fenómeno de desplazamiento, ya que durante la misma se puede beneficiar económica o socialmente, esto influye en que haya más mano de obra y ayuda en el desarrollo de una sociedad diversa $y$ sin discriminaciones.

\section{ABSTRACT}

The research studies of urban planning scenarios in the context of entrepreneurial productivity and competitiveness, in the city of Bogota, set the bases for the analysis, formulation and management of public policies for the population affected by local displacement.

This article aims at identifying the urban impact and formulation of migration public policies which are applied with a regulation effect. It is very important to take them into account for its best development and impact as this displaced population that lives in the city not always contribute to their fulfillment and application. This leads to identify and formulate the best public policies to follow up these displaced people that have the right to live in the city. Some of them probably don't want to exert a negative influence, but it is important to identify them to keep a social order.

A developing city looks for the best planning, permanently adapting and evolving in regard with the displacement phenomenon as it can bring economic and social benefits. This may lead to a more demand of hand work and it helps to the development of a more diverse society without signs of discrimination.

\section{Palabras clave}

\author{
Política \\ Migración \\ Emprendimiento \\ Productividad \\ Desplazamiento
}

Keywords

Policy

Migration

Entrepreneurship

Productivity

Displacement 


\section{RESUMÉÉ}

L'étude des scénarios de planification urbaine dans le contexte de productivité et de compétitivité des entreprises sert de support à l'analyse, à la formulation et à la prise de décisions des pouvoirs publiques pour associer les populations victimes de déplacements forcés aux politiques publiques. L'article cherche à identifier l'impact urbain et la formulation de politiques publiques migratoires permettant une régulation des populations. Les personnes déplacées présentes dans la ville de Bogotá ne contribuent pas toujours positivement au développement urbain, ce qui rend l'efficacité des politiques publiques mises en place peu efficaces et peu respectées. Nombre des déplacés ne souhaitent cependant pas nuire au fonctionnement de l'espace urbain, il est pour cela important et nécessaire de connaître et d'identifier ces individus pour maintenir l'ordre dans la société. Lors de son développement, la ville est soumise à la planification de l'administration publique qui s'adapte et évolue en fonction du phénomène du déplacement urbain avec pour objectif de contribuer à l'évolution d'une société diversifiée et sans discrimination.

\section{RESUMO}

$O$ estudo dos cenários de planejamento urbano no contexto da produtividade e a competitividade empresarial, para o caso da cidade de Bogotá, servem de suporte para a análise, a formulação e o direcionamento das políticas públicas que permitam envolver a população que sofre um deslocamento interno.

O artigo procura identificar o impacto urbano e a formulação das políticas públicas migratórias que se aplicam como efeito de regulação; é importante tê-las em conta para o bom funcionamento e desenvolvimento do mesmo posto que as pessoas deslocadas, que estão presentes na cidade, não sempre contribuem positivamente ao desenvolvimento urbano e isto faz que a efetividade das políticas públicas implementadas não sempre se cumpra ou se implemente na sua totalidade. Isso convida a que se formulem e identifiquem as políticas públicas para estabelecer um controle das pessoas que têm o direito de habitar.

Pode que muitas destas pessoas não queiram influir negativamente e passar desapercebidas, no entanto, é importante e necessário reconhecê-las e identificá-las para manter uma ordem na sociedade.

A cidade em desenvolvimento em alguns casos procura o planejamento, se adapta e evolui com o fenômeno do deslocamento, já que durante o mesmo se pode beneficiar econômica ou socialmente, isso influi em que haja mais mão-de-obra e ajuda no desenvolvimento de uma sociedade diversa e sem discriminações
Mots clefs

\author{
Politique \\ Migration \\ Entrepreneuriat \\ Productivité \\ Déplacement
}

Palavras-chave

\author{
Política \\ Migração \\ Empreendimento \\ Produtividade \\ Deslocamento
}




\section{DESPLAZAMIENTO A LA CIUDAD}

$\mathbf{L}$ a violencia que se ha generado en Colombia en las últimas décadas, ha causado daño a la[...] población, viéndose afectada desde lo rural a lo urbano y viceversa (Ceballos, 2010). La magnitud del impacto en el territorio, trae consecuencias especialmente en la sobresaturación demográfica de los perímetros urbanos o en espacios informales,

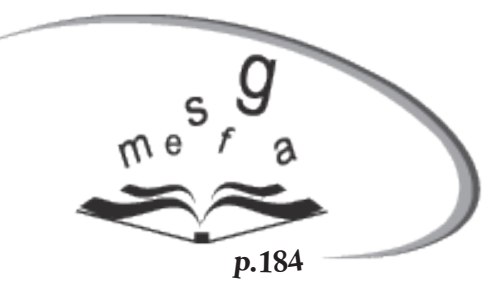
públicas coherentes a las necesidades humanas, urbanas y de la lógica de la planeación.

Es así como en Bogotá y especialmente en algunas localidades ${ }^{1}$, se sufre territorialmente a causa de este flagelo; al ser la capital de Colombia, se convierte en el principal destino por parte de los [...] desplazados (Peña, 2003) motivados por nuevas oportunidades, proyectos de vida, etc. Este flujo migratorio desarrolla una actividad que va desde la adaptación al territorio urbano y su trasformación, replicando instintivamente en las características rurales.

Figura 1. Esquema de investigación

\section{Objetivos}

Comprender los comportamientos existentes que evalúan el impacto social y económico del desplazamiento a la Capital en la implementación de políticas públicas.

\section{Pregunta}

¿De qué manera el desplazamiento influye en las concepciones desarrolladas en la ciudad y si este fenómeno incide en la productividad urbana?

¿Qué ventajas competitivas ofrecen las políticas públicas en torno a los escenarios de hábitat urbano para el caso de la ciudad de Bogotá en la construcción del tejido social y redes empresariales?

Fuente. Ocampo, 2013.

\footnotetext{
${ }^{1}$ Bogotá se subdivide en 20 localidades y en estas se agrupan más de 1.200 barrios que hay en el casco urbano de la ciudad. Debido a sus diferencias y problemáticas particulares, cada localidad cuenta con una alcaldía local, que se convierte en centro de contacto más cercano del ciudadano con la administración distrital (Bogotá cómo vamos, 2013).

2 El programa "Bogotá cómo vamos" hace seguimiento a los cambios en la calidad de vida de la ciudad el cual maneja una batería de indicadores técnicos y de percepción que permiten tener una mirada global de los aspectos que inciden en las condiciones de vida de los bogotanos.
}

La principal modificación del paisaje urbano por parte de los habitantes desplazados, es la necesidad de un techo; la vivienda se convierte en la prioridad bajo condiciones de mayor seguridad para sus familias, así sea con deficiente infraestructura en términos de servicios públicos que en su gran mayoría carecen o son compartidos por diferentes familias o asentamientos, la estructura de vivienda con las condiciones mínimas de habitabilidad genera adicionalmente un problema estético ante la imposibilidad de cumplir con una política pública.

Según los datos del programa: "Bogotá cómo vamos", presentado el jueves 4 de julio de 2013 en la Cámara de Comercio de Bogotá2, la importancia de Bogotá en el contexto nacional es indiscutible. La ciudad concentra el $16 \%$ de la población del país y aporta el $24,5 \%$ del Producto Interno Bruto Nacional (PIB). El crecimiento económico sostenido de los últimos 5 años, ha permitido que los 7'571.345 de bogotanos disfruten de un PIB per cápita de $\$ 12.000$ dólares.

Este panorama hace atractiva la ciudad y se convierte en el principal destino en el caso de la población afectada por el flagelo.

La favorable situación económica y las políticas sociales, han permitido que la pobreza haya bajado desde 13,1\% en 2011 hasta el 11,6\% en 2012 (Bogotá cómo vamos, 2012).

A pesar del resultado de percepción, las personas, dependiendo del lugar de origen, suelen dispersarse, pero inicialmente su primera opción responde a la entrada a la ciudad con la tendencia de tener un pie cerca de su lugar de origen. Concentran sus actividades en las localidades más pobres afectando el entorno 
y las unidades territoriales vecinales ya que estas se encuentran en el perímetro urbano con las condiciones ideales para establecer relaciones sociales, económicas y productivas, así estas sean instintivas o de réplica según los referentes del entorno.
Esta tendencia enmarca situaciones que las estadísticas no miden como es, la dificultad de adaptación y la apropiación del lugar del entorno con el agravante de los recursos limitados por parte de las organizaciones públicas para la ayuda de las necesidades básicas insatisfechas, con el rechazo de sectores de la población y del entorno consolidado. Esto responde a la competencia por el territorio y sus necesidades de abastecimiento.

\section{CONFLICTO Y TERRITORIO}

E I territorio que contiene la ciudad capital, termina aprendiendo cada día y sufriendo con base en el contexto del conflicto armado interno que vive el país. Paradójicamente, sin ser el centro del conflicto, sufre notablemente las consecuencias, especialmente las localidades donde se asientan evidencia el incremento

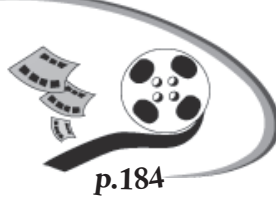
de la población; de ser una ciudad de 350 habitantes en los años 50, pasó a ser una ciudad de siete millones según los datos del Departamento Administrativo Nacional de Estadística (DANE).

Esto conlleva a un incremento de [...] violencia, desigualdad social (DISTRITAL, 2004) y se corrobora en la información suministrada por la Policía Metropolitana de Bogotá ${ }^{3}$ [...] en el 2012 fueron incautadas 2.147 armas de fuego por el delito de porte ilegal. El 54,4\% de las armas incautadas en la ciudad, se concentraron en las localidades de Ciudad Bolívar ${ }^{4}(13,7 \%)$ territorio en donde se concentra la mayor población víctima de la violencia (Policía Metropolitana de Bogotá).

Este fenómeno no es ajeno para otras localidades ${ }^{5}$ que se encuentran en situaciones similares a la Bogotá.
Ciudad Bolívar, Rafael Uribe Uribe, Usme, Bosa y Usaquén, son las de mayor ocupación informal de la comunidad desplazada en la ciudad.

Esta situación no sólo se ve reflejada en los temas de violencia, sino también en una escala indeterminada. El problema se concentra en el hábitat; se encontró que (...) la Secretaría Distrital del Hábitat, a través de la Subsecretaría de Inspección, Vigilancia y Control de Vivienda, viene realizando visitas a las zonas susceptibles de ocupación ilegal que se encuentran en 13 localidades de Bogotá y encontró 16.801 ocupaciones ilegales en la ciudad (Hábitat, 2013).

A pesar del esfuerzo que hacen las entidades públicas, la dinámica territorial está sujeta a las estadísticas que muestran las evidencias, pero que en su gran mayoría, es poco medible por las dinámicas mismas del desplazamiento.

Los datos que da la Secretaría de Hábitat de Bogotá, muestran que luego de una inspección a la localidad de Ciudad Bolívar, se halló:

\footnotetext{
3 El 26 de enero de 2012, se expidió la Resolución 001 por medio de la cual se suspende de manera general y por un tiempo determinado, el porte de armas de fuego en la ciudad, con el objetivo de desincentivar su uso y disminuir los índices de criminalidad.

4 Localidad No. 19 del Distrito Capital de Bogotá. La localidad está declarada por la autoridades distritales como zona roja.

5 Bogotá se subdivide en 20 localidades y en estas se agrupan más de 1.200 barrios que hay en el casco urbano de la ciudad. Debido a sus diferencias y problemáticas particulares, cada localidad cuenta con una alcaldía local, que se convierte en centro de contacto más cercano del ciudadano con la administración distrital.
} 
Tabla 1. Predios con el mayor índice de ocupaciones ilegales.

\begin{tabular}{|c|l|l|}
\hline $\begin{array}{c}\text { PREDIOS } \\
\text { CIUDAD BOLIVAR }\end{array}$ & \multicolumn{1}{|c|}{ OCUPACIONES } & \multicolumn{1}{c|}{ DISTRIBUCIÓN Y CARACTERÍSTICAS } \\
\hline 6.531 & ocupaciones ilegales & 34 zonas visitadas (402 hectáreas) \\
\hline 3.093 & ocupaciones ilegales están consolidadas & $\begin{array}{l}\text { Es decir, son hogares que ya cuentan con una } \\
\text { infraestructura definida (columnas, planchas } \\
\text { o material de construcción determinado). }\end{array}$ \\
\hline $\begin{array}{c}\text { PREDIOS EN } \\
\text { RAFAEL URIBE URIBE }\end{array}$ & OCUPACIONES & DISTRIBUCIÓN Y CARACTERÍSTICAS \\
\hline & ocupaciones ilegales con 2.510 & $\begin{array}{l}\text { 65\% corresponden a lotes donde se ha } \\
\text { cercado arbitrariamente el terreno }\end{array}$ \\
\hline
\end{tabular}

Fuente. Bogotá cómo vamos, (2013).

Le siguen: (Tabla 2)

Tabla 2. Localidades con ocupaciones ilegales.

\begin{tabular}{|l|l|}
\hline Usme & 1.71 ocupaciones ilegales \\
\hline Bosa & 1.562 ocupaciones ilegales \\
\hline Usaquén & 1.437 ocupaciones ilegales \\
\hline Kennedy & 722 ocupaciones ilegales \\
\hline Suba & 696 ocupaciones ilegales \\
\hline Tunjuelito & Menos ocupaciones ilegales 47 \\
\hline Engativá & Menos ocupaciones ilegales 57 \\
\hline
\end{tabular}

Fuente. Secretaría de Hábitat en Bogotá, (2013).

Es importante anotar que el tema se discute en las últimas décadas dentro de los puntos de las campañas y presentación de los planes de gobierno, anexando las posibles soluciones o alternativas sociales y teniendo en cuenta la situación que el país vive. Lo anterior se orienta a la formulación de políticas públicas que no siempre son continuas y eficientes, sobre todo tratándose a población vulnerable.

Para la ciudad, el fenómeno del desplazamiento constituye el elemento más visible. De acuerdo con los registros de la Consultoría para los Derechos Humanos y el Desplazamiento, el estimativo de la población desplazada (ACNUR), que datan de 1980 hasta agosto de 2012, supera los cinco millones de personas (Tabla 3).
Tabla 3. Indicadores de desplazados

\begin{tabular}{|l|l|}
\hline $\begin{array}{l}\text { Según la Red de Solidaridad } \\
\text { Social - RSS }\end{array}$ & $\begin{array}{l}\text { El 23\% del total de la po- } \\
\text { blación es desplazada en el } \\
\text { país }\end{array}$ \\
\hline $\begin{array}{l}\text { Sistema de Información de la } \\
\text { Unidad de Atención Integral } \\
\text { a la Población Desplazada } 13.718 \text { hogares corres- } \\
\text {-UAID-, del Distrito Capital } \\
\text { según la UAID, la Contraloría } \\
\text { Distrital }\end{array}$ & \\
\hline & $\begin{array}{l}\text { ponden aproximadamente a } \\
\end{array}$ \\
& $\begin{array}{l}\text { El } 48.9 \% \text { personas, } \\
\text { plazadas que llegan a Bogotá } \\
\text { están registrados como tal. }\end{array}$ \\
\hline
\end{tabular}

Fuente. Ocampo,2013.

Es así como el distrito recibe entre el 16\% y $18 \%$ de la población desplazada del país, los sitios de procedencia que se menciona en la siguiente tabla, según investigaciones de CODHES, vienen directamente de los departamentos que a continuación se relacionan (Tabla 4).

Tabla 4. Lugares de procedencia de los desplazados

\begin{tabular}{|l|l|}
\hline $\begin{array}{l}\text { Meta, Guaviare y } \\
\text { Caquetá }\end{array}$ & Años 90 \\
\hline \multicolumn{2}{|l|}{ Antioquia, Córdoba y Chocó } \\
\hline Antioquia, Córdoba y Chocó \\
\hline Tolima, Huila y Cundinamarca \\
\hline UAID & $\begin{array}{l}\text { Las familias atendidas provienen } \\
\text { de Tolima, Meta, Cundinamarca, } \\
\text { Antioquia y Caquetá }\end{array}$ \\
\hline
\end{tabular}

Fuente. Corporación Nuevo Arcoíris, 2008. 


\section{DIFICULTADES EN LA IMPLEMENTACIÓN DE LAS POLÍTICAS PÚBLICAS PARA DESPLAZADOS}

Lian os múltiples problemas que enfrenta un territorio y la implementación de políticas públicas eficientes y eficaces para atender el desplazamiento, hacen que cualquier decisión, propuesta o ejecución se vea en aprietos. El hecho de no tener claros los registros de la población desplazada, coincide con las apreciaciones de Lozano (2012), [...], en una desacertada información es muy difícil implementar una metodología unificada para la cobertura, recolección,

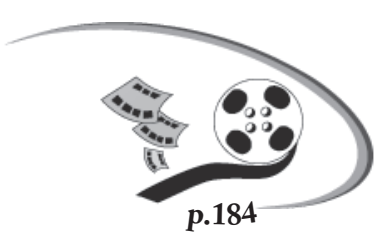
procesamiento, consolidación y verificación de la información sobre el número de desplazados". En este mismo orden de ideas, es oportuno pensar las implicaciones en la seguridad y convivencia durante el posconflicto, ya que de algún modo, si los diálogos de paz que adelanta el Gobierno Nacional y la propuesta de justicia transicional ${ }^{6}$ concluyen de manera positiva, la desmovilización y reintegración de los grupos guerrilleros podría afectar la seguridad de los entornos urbanos.

La ciudad, las empresas, los actores públicos, privados y comunitarios, deben estar preparados e indicar su participación en este tema. Es importante establecer su papel en estos procesos y evaluar los resultados de la experiencia que se tuvo a partir del año 2005, con la reinserción de los grupos paramilitares desmovilizados. (Bogotá Cómo Vamos, 2012). Lo anterior, invita a preguntarse cuál será la fase inicial de la planeación urbana en el contexto de productividad y la competitividad empresarial, involucrando la población desplazada y si esta reflexión permite la formulación de un modelo conceptual y metodológico desde las políticas públicas urbanas. Previendo esta situación, en la investigación se estructuró la pregunta (Figura 2).

6 La justicia transicional, es el conjunto de medidas judiciales y políticas que diversos países han utilizado como reparación por las violaciones masivas de derechos humanos. Entre ellas figuran las acciones penales, las comisiones de la verdad, los programas de reparación y diversas reformas institucionales. 
Figura 2. Investigación "La planeación urbana en el contexto de la productividad y Competitividad empresarial política urbana para desplazados 2013".

Fase uno: la planeación urbana en el contexto de la productividad y la competitividad empresarial

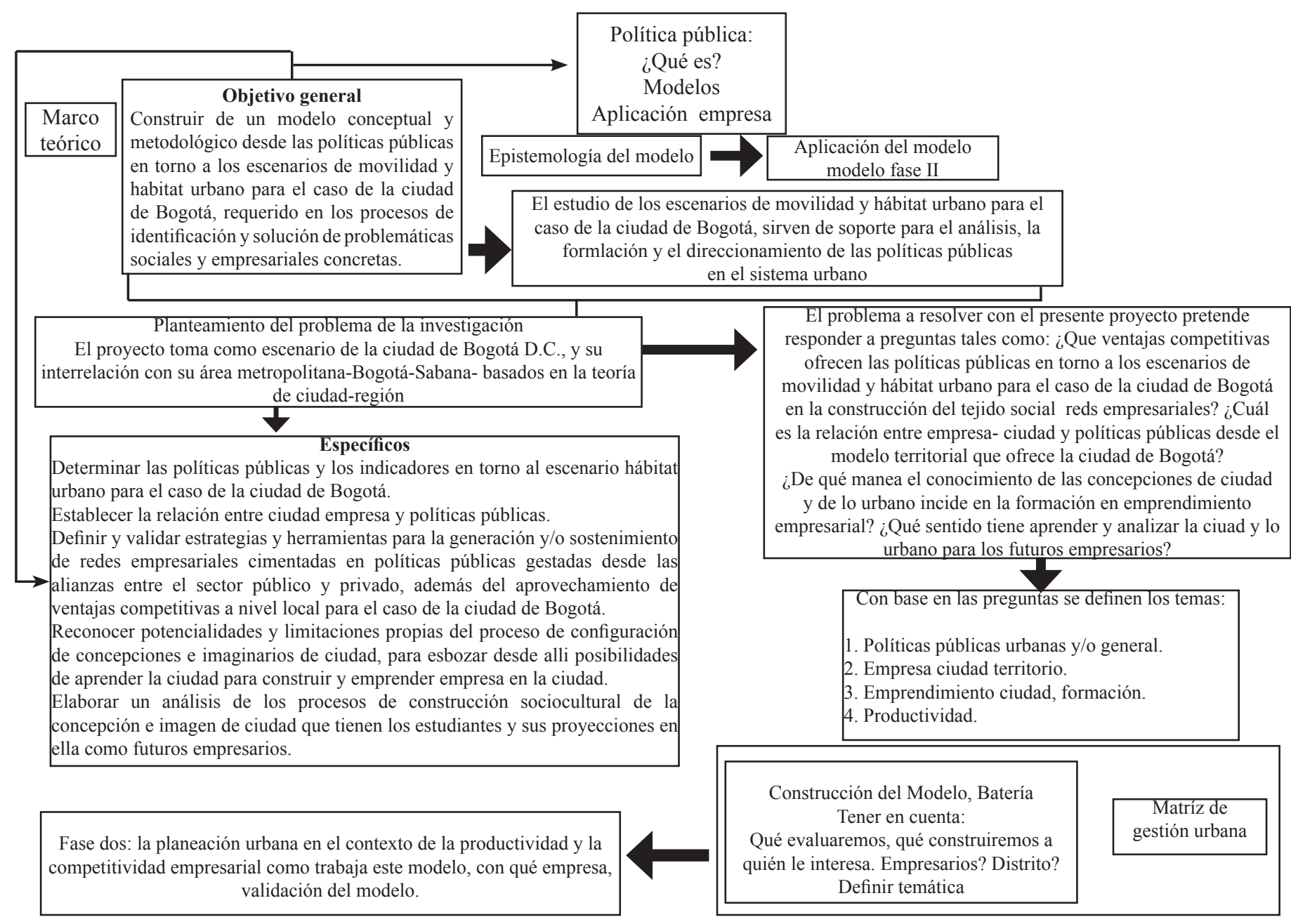

Fuente. Ocampo, Cely, González, 2013.

De este esquema se plantea una matriz de gestión que se pondrá en marcha en la segunda fase de la investigación, invitando a la reflexión e identificación de los asuntos que están por fuera del alcance de un gobierno territorial y que corresponden a la toma de decisiones y acciones ideales en la implementación de las políticas públicas involucrando los actores en confrontación.

Esta matriz de gestión se concibe entendiendo las características de los asentamientos humanos que están viviendo tiempos de intensa discusión, éstas han tomado diez años desde que se pone en marcha y ejecución el
Plan de Ordenamiento Territorial (POT), formulado con la ley 388 de 1997.

Aún se debate acerca de cómo debe ser la planificación urbana, si es inclusiva o si el interés general prevalece sobre el particular; aquí es evidente que no se cumple este principio Constitucional, pues el interés particular prima sobre el general siendo característico desde la llegada de los españoles donde el territorio pasa a ser propiedad privada.

Desde ese entonces, la formulación de las políticas urbanas están encaminadas a implementar las acciones 
de gobierno que buscan dar respuestas a las diversas demandas de la sociedad, con el propósito de definir objetivos prioritarios, lineamientos y estándares de cumplimiento y provisión que deben ser alcanzados para asegurar una adecuada prestación de servicios y el normal desarrollo de las actividades del Estado (Figura 3).

\section{Figura 3.}

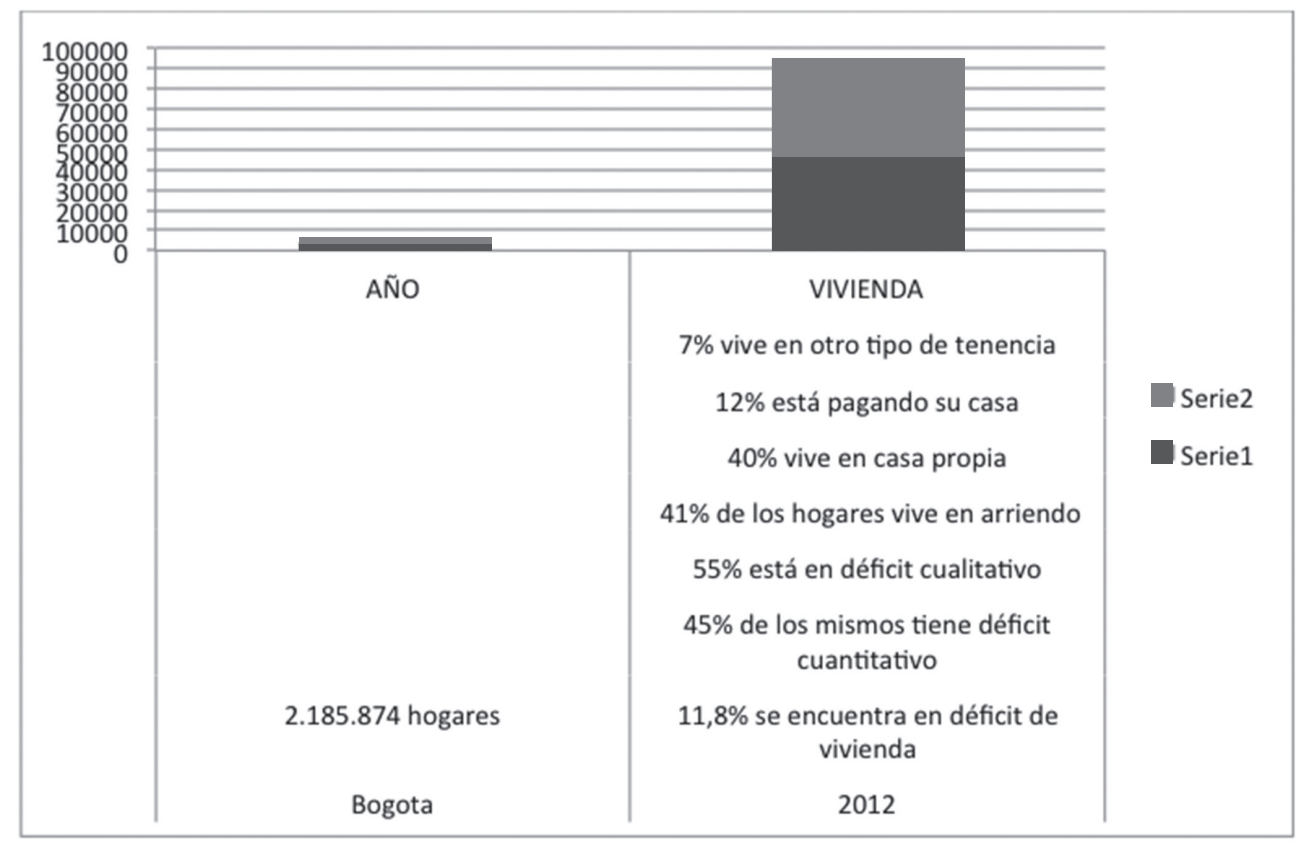

Fuente. Elaboración propia del autor, a partir de "Bogotá cómo vamos", (2012).

La ciudad capital, como receptora de población desplazada, incurre en una serie de costos relacionados con aspectos que reflejan la problemática socioeconómica actual de las familias asentadas en Bogotá, (Tabla 5).

Tabla 5. Costos problemática socioeconómica de familias a sentadas en Bogotá.

\begin{tabular}{|c|l|}
\hline Empleo & $\begin{array}{l}\text { Población económicamente activa bajo situación de desempleo o tasa de desempleo: 51.8\% } \\
\text { (15.378). }\end{array}$ \\
\hline Salud & Familias no afiliadas al sistema de salud: 22\% (23.927) \\
\hline Educación & Población menor de 18 años sin cupos escolares: 49\% (125.644) \\
\hline Vivienda & $\begin{array}{l}\text { Familias que no poseen vivienda actualmente y antes poseían, o pagan arriendo en la actualidad: } \\
66 \% \text { (73.751) }\end{array}$ \\
\hline Servicios Públicos & $\begin{array}{l}\text { Familias con déficit en energía: 5\% (5.676), acueducto: 8\% (8.338), aseo: 10\% (11.018), } \\
\text { alcantarillado: 10\% (10.683), teléfono: 19\% (21533) y gas: 32\% (35.379); promedio de familias } \\
\text { con déficit en todos los servicios: 13\% (15.437) }\end{array}$ \\
\hline Gastos Operacionales & Personal y funcionamiento de la organización institucional para atender esta problemática \\
\hline $\begin{array}{c}\text { Invasión del espacio } \\
\text { público 44,7\% }\end{array}$ & $\begin{array}{l}\text { La ocupación de vías, andenes, parques o zonas verdes, puentes y barrios, entre otros. Otros } \\
\text { aspectos }\end{array}$ \\
\hline $\begin{array}{c}\text { Incremento del } \\
\text { desempleo }\end{array}$ & $\begin{array}{l}\text { (38,7\%), la inseguridad (34,1\%), el empleo informal (27,1\%) y los asentamientos subnormales } \\
(15,5 \%) .\end{array}$ \\
\hline
\end{tabular}

Fuente. Elaboración propia del autor, a partir de "Bogotá cómo vamos", (2012) 
La información anterior muestra la sensación negativa del fenómeno del desplazamiento en la ciudad y una imagen también negativa de sus efectos sobre la ciudad en asuntos cruciales de interés de la ciudadanía, como es el espacio público y la seguridad. Pero lo más importante, es la probable fuente de conflictos que constituye la llegada de nuevos migrantes a la ciudad, que compite especialmente con los estratos más pobres por la ocupación laboral.

\subsection{Cuestión de norma}

El interés de tener una norma clara, objetiva, equitativa, incluyente, entre otras buenas intenciones, ha motivado una ardua labor a la Corte Constitucional colombiana que en múltiples oportunidades establece parámetros referentes al caso de estudio. Durante esta investigación se encontró que mediante la sentencia [...] T-025 del 22 de enero de 2004, al pronunciarse sobre 109 expedientes, fueron 1.150 los núcleos familiares de diferentes regiones del país los que interpusieron acciones de tutela (Constitucional, 2004).

No obstante, esta visión esta inmensamente ligada a las opiniones base de identificación de modelos de las políticas públicas planteadas en el documento de la ACNUR. La Política Pública de Prevención y Atención al Desplazamiento Interno Forzado en Colombia 2002-2004, identifica que aún cuando reconoce avances importantes en el incremento de recursos nacionales asignados para la política respectiva, plantea la responsabilidad y el esfuerzo que se avecina, donde se deben involucrar los actores internacionales y la equitativa distribución de los esfuerzos económicos (Figura 4). 
Figura 4. Leyes generales sobre el desplazamiento
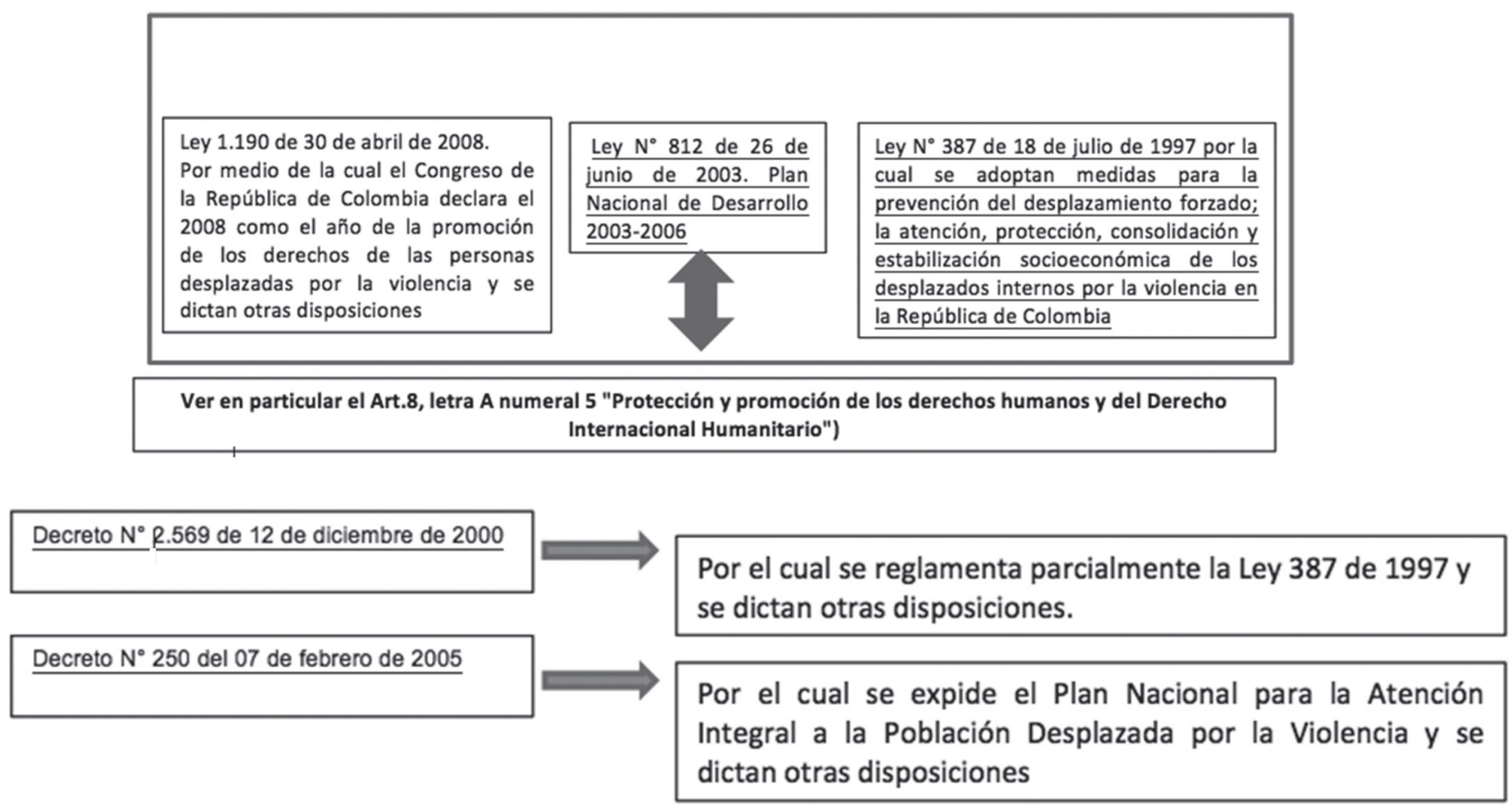

\begin{tabular}{|c|c|}
\hline Decreto $\mathrm{N}^{\circ} 1.547$ de 19 de agosto de 1999 & $\begin{array}{l}\text { Por el cual se traslada la administración integral del Fondo } \\
\text { Nacional para la Atención a la Población Desplazada por la } \\
\text { Violencia }\end{array}$ \\
\hline Decreto $N^{\circ} 173$ de 26 de enero de 1998 & $\begin{array}{l}\text { Por el cual se adopta el Plan Nacional para la Atención } \\
\text { Integral a la Población Desplazada por la Violencia }\end{array}$ \\
\hline Decreto $\mathrm{N}^{\circ} 976$ del 7 de abril de 1997. & $\begin{array}{l}\text { Por el cual se reglamenta el artículo } 70 \text { del Decreto-ley } 919 \\
\text { de } 1989\end{array}$ \\
\hline Decreto $N^{\circ} 489$ de 11 de marzo de 1999 & Por el cual se asigna una función \\
\hline Decreto $N^{\circ} 501$ del 13 de marzo de 1998. & $\begin{array}{l}\text { Por el cual se establece la organización y funcionamiento } \\
\text { del Fondo Nacional para la Atención Integral a la Población } \\
\text { Desplazada por la Violencia y se dictan otras disposiciones. }\end{array}$ \\
\hline Acuerdo 02 de 1998 & El que precisa las normas para la atención integra. \\
\hline Decreto 624 de 1998 e 1998 & $\begin{array}{l}\text { Establece la creación y funcionamiento del Consejo Distrital } \\
\text { de Atención de la Población Desplazada, constituyen un } \\
\text { legado normativo propicio para el desarrollo de la política. }\end{array}$ \\
\hline
\end{tabular}

Fuente. Ocampo, a partir de Congreso de la República; Consejo de Bogotá, 2013. 
Tabla 6. Entidades que manejan el tema de desplazados

\begin{tabular}{|c|c|}
\hline ACNUR & Alto Comisionado de las Naciones Unidas para los Refugiados \\
\hline AHE & Atención Humanitaria de Emergencia \\
\hline CICR & Comité Internacional de la Cruz Roja \\
\hline Conpes & Consejo Económico y Social \\
\hline CNAIPD & Consejo Nacional para la Atención Integral a la Población Desplazada por la Violencia \\
\hline DIH & Derecho internacional Humanitario \\
\hline DNP & Departamento Nacional de Planeación \\
\hline FNR & Fondo Nacional de Regalías \\
\hline ICA & El impuesto de Industria y Comercio \\
\hline ICBF & Instituto Colombiano de Bienestar Familiar \\
\hline IDP & Desplazados internos (Internally Displaced Person) \\
\hline IGAC & Instituto Geográfico Agustín Codazzi \\
\hline INCODER & Instituto Colombiano para el Desarrollo Rural \\
\hline JUNTOS & la Red para la Superación de la Pobreza Extrema \\
\hline MEN & Ministerio de Educación Nacional \\
\hline MIJ & Ministerio del Interior y Justicia \\
\hline ONGs & Organizaciones no gubernamentales \\
\hline PIU & Planes Integrales Únicos \\
\hline RSG & $\begin{array}{l}\text { Representante del Secretario-General de las Naciones Unidas sobre los Derechos Humanos de los } \\
\text { Desplazados Internos }\end{array}$ \\
\hline RUP & Registro Único de Predios Abandonados del INCODER \\
\hline RUPD & Registro Único de Población Desplazada \\
\hline RUT & Registro Único Tributario \\
\hline SENA & Servicio Nacional de Aprendizaje \\
\hline SGPP & Sistema General de Participaciones \\
\hline SGSSS & Sistema General de la Seguridad Social en Salud \\
\hline CODHES & Consultoría para los Derechos Humanos \\
\hline \multirow[t]{6}{*}{ SDIS } & Secretaria Distrital de Integración Social \\
\hline & Secretaria Distrital de Salud \\
\hline & Personería de Bogotá \\
\hline & Secretaria Distrital de Gobierno \\
\hline & Secretaria Distrital de Hábitat \\
\hline & Secretaria Distrital de Desarrollo Económico \\
\hline \multirow[t]{2}{*}{ IPES } & Instituto para la Economía Social \\
\hline & Secretaria Distrital de Educación \\
\hline UAO & Unidades de Atención y Orientación a la población desplazada \\
\hline
\end{tabular}

Fuente. Elaboración propia del autor, (sf). 
Tabla 7. Actividades de socialización de la comunidad

\begin{tabular}{|l|}
\hline Familias en Acción \\
\hline Eje Social \\
\hline Eje Urbano Regional \\
\hline Eje de Reconciliación \\
\hline Derechos Humanos para todas y todos \\
\hline $\begin{array}{l}\text { Atención a población en condiciones de desplazamiento forzado, desmovilización o vulnerabilidad frente a la violencia, con perspectiva de } \\
\text { Derechos Humanos y de Derecho Internacional Humanitario }\end{array}$ \\
\hline Restitución de Activos \\
\hline Sistema de Registro Único \\
\hline Programas de generación de ingresos (Capacitación laboral, Microcréditos y proyectos productivos) \\
\hline Educación \\
\hline Salud
\end{tabular}

Fuente. Ocampo, 2013.

Investigación La planeación urbana en el contexto de la productividad y Competitividad empresarial política urbana para desplazados 2013.

El programa "Bogotá cómo vamos" reconoce los avances en las acciones del Distrito para responder al problema del desplazamiento. Destaca que bajo los principios de subsidiaridad, complementariedad, descentralización y concurrencia, la Administración
Distrital ha configurado, desde la Secretaría de Gobierno y en coordinación con la Red de Solidaridad Social, dispositivos institucionales como la Unidad de Atención Integral. Este documento tan importante de percepción, invita a la elaboración de preguntas que ayuden a generar acciones de evaluación de las políticas públicas como ¿Cuál es la claridad de las políticas públicas? Esta pregunta se hace a 200 personas, 100 hombres y 100 mujeres.

\section{Figura 5. Claridad de las políticas públicas}

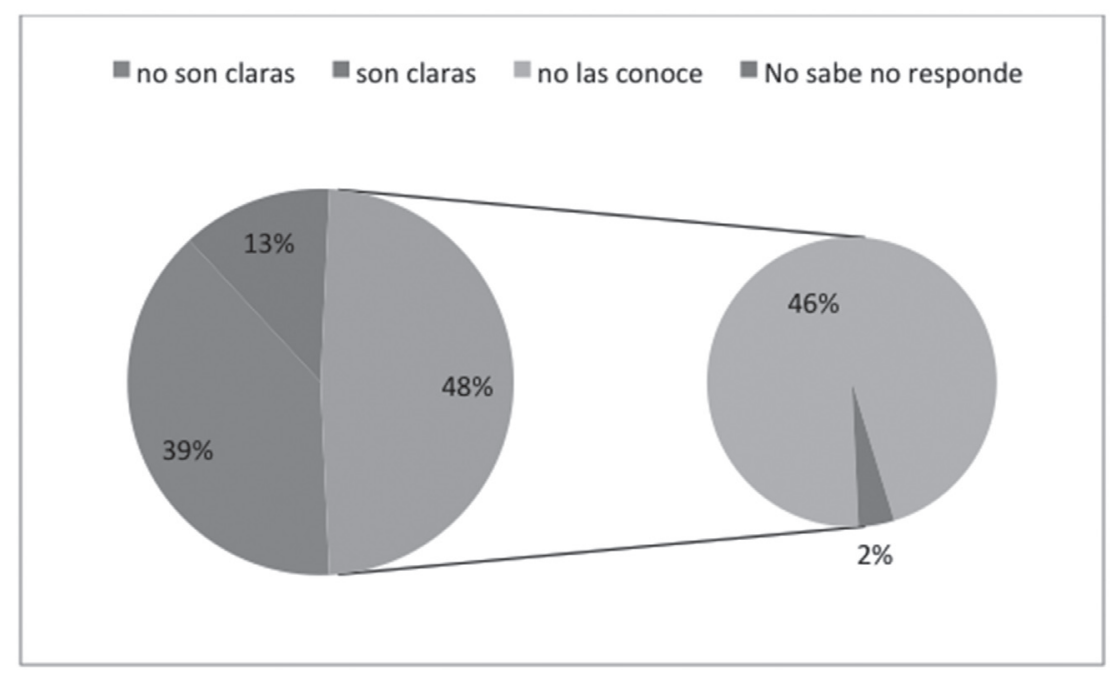

Fuente. Ocampo E., Velásquez, Perilla, 2013. 
En definitiva, el desconocimiento de las políticas públicas es muy alto, ya que corresponde al $46 \%$ de los encuestados; esto, indica que la eficiencia de las mismas se pueden poner en duda, sin embargo, es importante resaltar que un 39\% indicó que no son claras, lo que lleva a pensar que es motivada a percepción más no a conocimiento de las mismas.

Figura 6. ¿Cómo ha sentido en su cotidianidad el problema del desplazamiento forzado por la violencia en Bogotá?

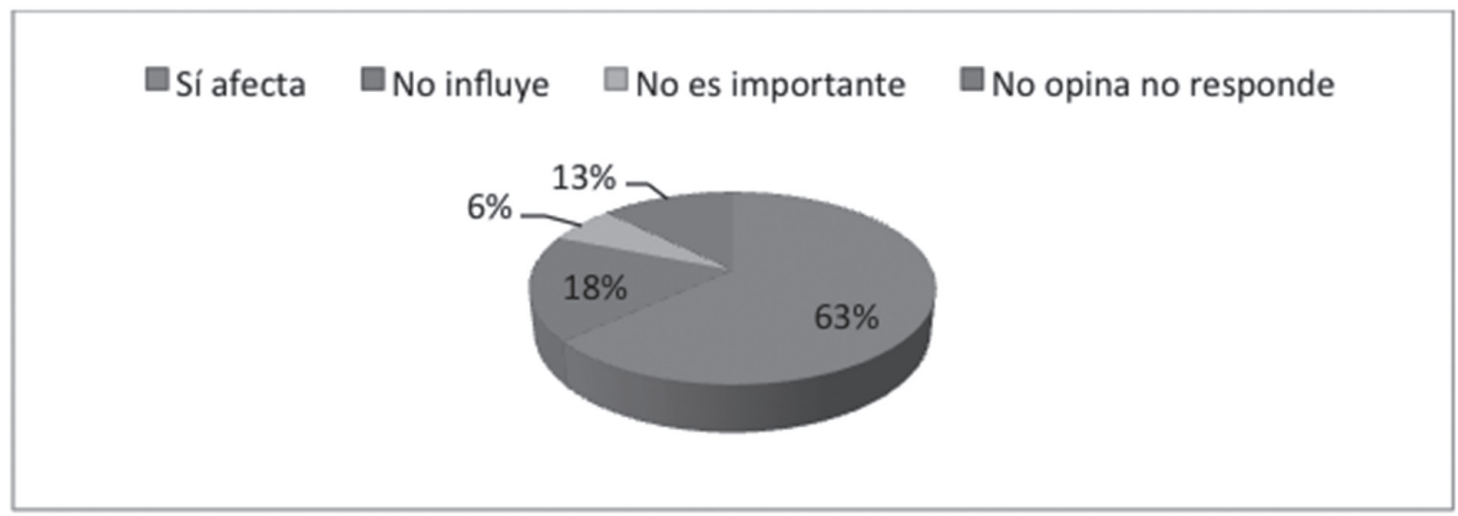

Fuente. Ocampo, Velásquez, 2013.

El $63 \%$ de las personas indica que se ven afectadas e indican que es poco eficiente; en especial, las mujeres coinciden en que el problema del desplazamiento forzado incide en la violencia. La información anterior muestra las opiniones tan dispersas frente a la violencia generada por este flagelo, que determinan la apropiación y la calidad de vida de sus habitantes. La acción ciudadana tiene también un papel significativo en este asunto, en cuanto ha sustituido o complementado la acción del Estado; muchas comunidades han construido sus propias soluciones, el trabajo colectivo lo permite.

Figura 7. ¿Cuáles son los principales problemas derivados de este fenómeno en su localidad o en su vecindad y si estos afectan la formulación de proyectos productivos?

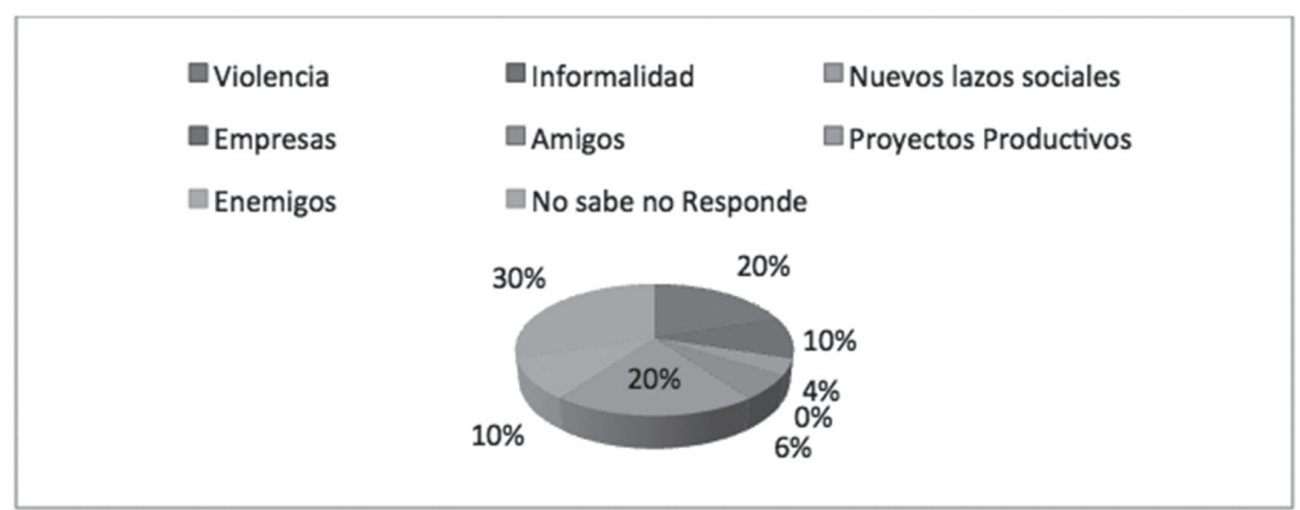

Fuente. Ocampo, Velásquez, 2013.

En esta pregunta, sorprende la mirada optimista y el deseo de salir adelante con la creación de proyectos. Estos están sujetos a la solidaridad, no obstante, se puede evidenciar la desconfianza que existe con la llegada de personas ajenas a la comunidad. El 4\% corresponde a los lazos sociales que influyen en la apropiación del territorio; este modelo de asociatividad, corresponde al necesario deseo de establecer redes sociales que se apoyen en situaciones de vulnerabilidad, lo que obliga a las políticas públicas a establecer soluciones en la formulación de proyectos y plantear nuevas formas de pensar e incorporar los comportamientos sociales. 
Figura 8. ¿Cómo hace una persona desplazada para acceder a servicios de salud?

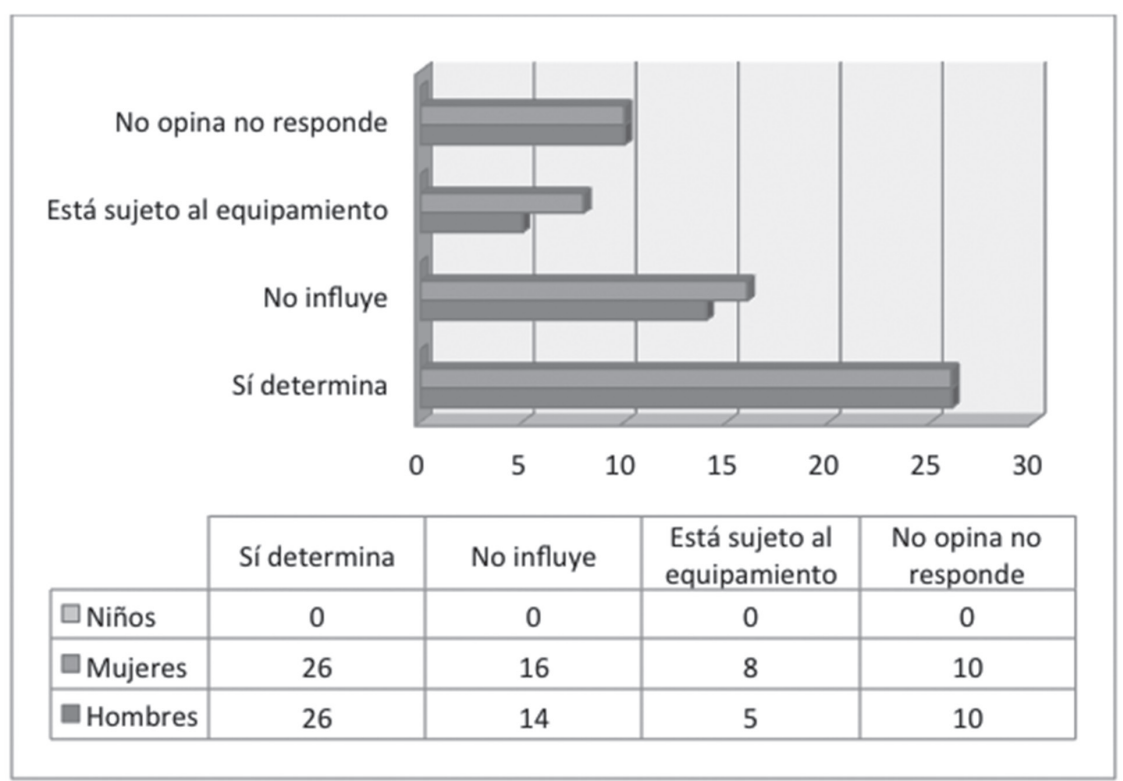

Fuente. Ocampo, Velásquez, 2013.

Con un empate se determina la relación de la existencia del equipamiento y el tipo de cobertura, preexiste el abandono y la falta de recursos lo que invita a la necesidad de implementar una política efectiva sin desconocer la situación de quien aspira a utilizar el servicio que por ley le corresponde. Esta situación no sólo consiste en adquirir un servicio eficiente sino además en incluir los tiempos. Sólo en una estructura adecuada se encuentra el complemento indispensable del sentido individual de acceder a la salud y es, en este forma, en el que debe pensarse y realizarse toda intervención destinada a mejorar la calidad de vida.

\section{Figura 9. ¿Qué determina el acceso a los a servicios de educación?}

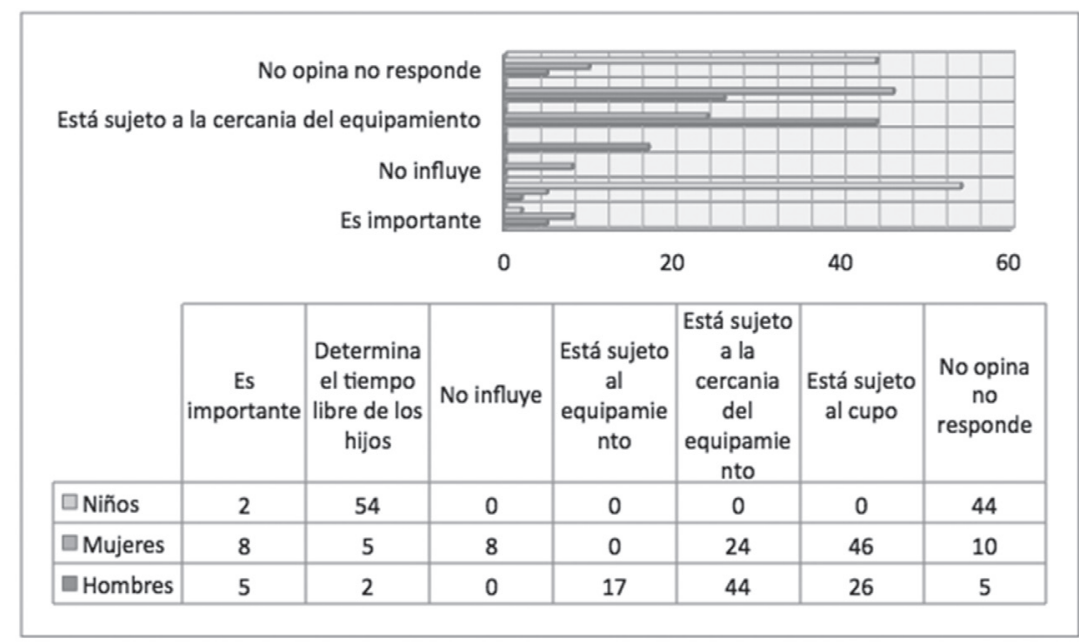

Fuente. Ocampo, Velásquez, 2013.

Con respecto al acceso a la educación, llama la atención cómo se estructura el tiempo con base en los servicios del Estado o distrito, dándole una prioridad a la existencia de los cupos; en segunda instancia, la cercanía al lugar de residencia casi que con un empate, pero siendo este de mayor valor para las mujeres que 
necesitan ocupar a sus hijos con la ilusión de salir adelante; casi que esta relación determina el éxito de la cobertura.

Se identifica que no importa la calidad o el estado de las instalaciones y la prestación del servicio, mientras cumpla con las condiciones mencionadas anteriormente. La falta de recursos económicos evidencia la necesidad de implementar una política efectiva, sin desconocer la situación de quien aspira a utilizar el servicio que por ley le corresponde. Esta situación no sólo consiste en adquirir un servicio eficiente, sino también tener en cuenta el tiempo requerido para este servicio. Sólo en una estructura adecuada se encuentra el complemento indispensable del sentido individual de acceder a la educación y es en esta vía, en la que debe pensarse y realizarse toda intervención destinada a mejorar la calidad de vida.

Figura 10. ¿Cuál es la imagen que se tiene en la localidad y entre sus vecinos sobre el desplazado?

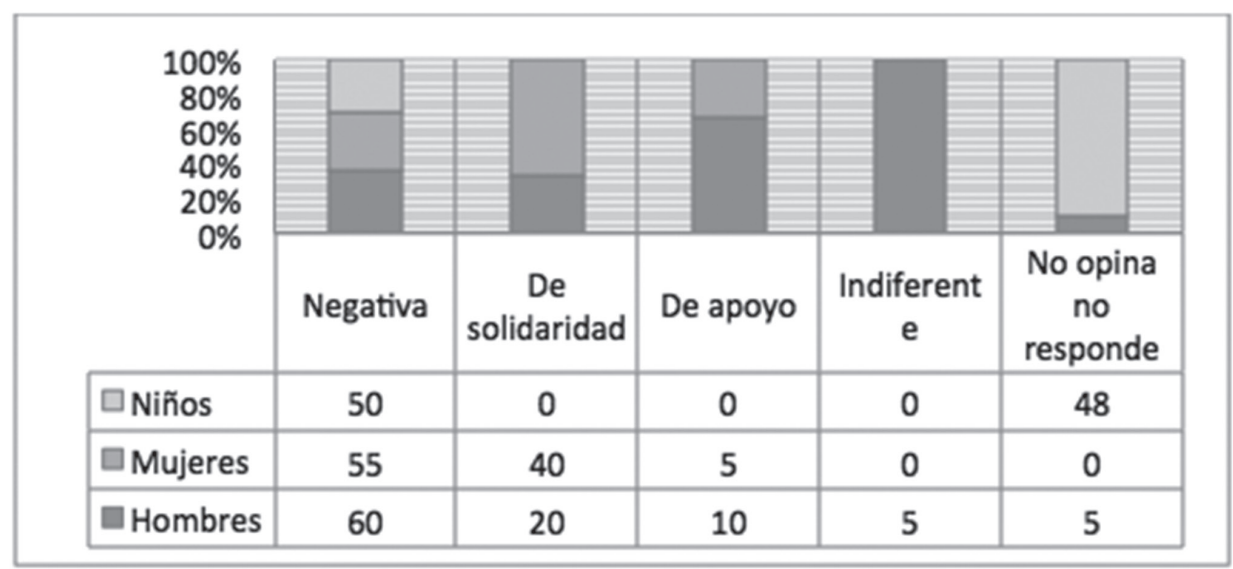

Fuente. Ocampo, Velásquez, 2013.

Es evidente que el impacto que genera la llegada de individuos ajenos a los lugares consolidados en los asentamientos urbanos, están determinados por el impacto de la percepción que la comunidad genere. Predomina la imagen negativa para las mujeres que se ven afectadas en la influencia y formación en su hogar, lo consideran un ejemplo negativo para su familia y comunidad; para los hombres, es igual una forma de justificar su participación según los indicadores encontrados; para algunos niños, es indiferente, puesto que esto está asociado a las actividades recreativas existentes en su unidad territorial, es importante resaltar que no se pueden identificar las potencialidades empresariales y competitivas del espacio, sin tener en cuenta la realidad del uso.

Figura 11. El asentamiento informal involucra las actividades cotidianas del lugar

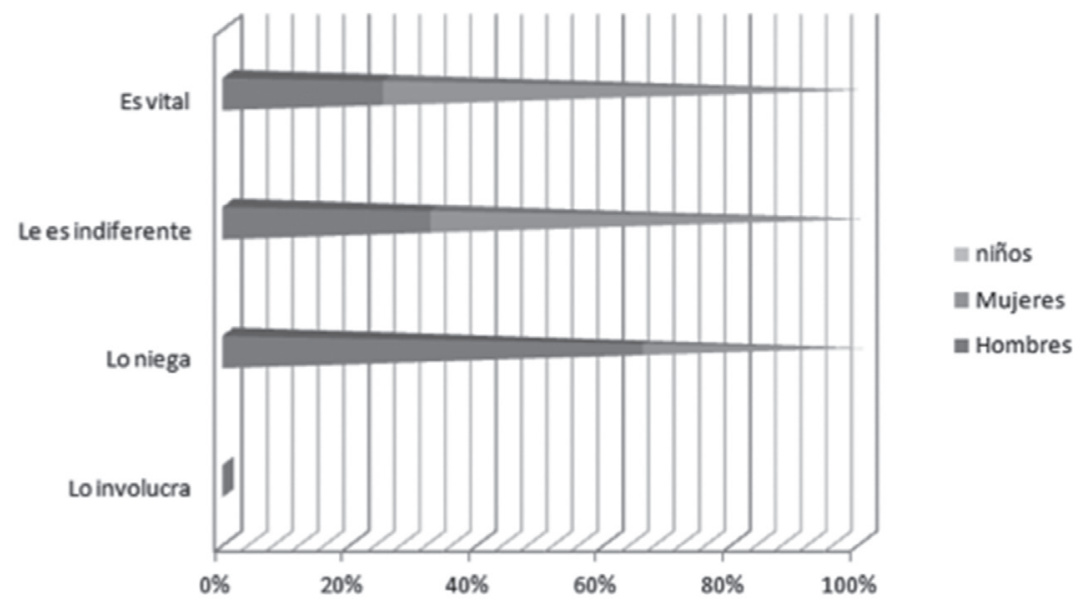

Fuente. Ocampo, Velásquez, 2013. 
En los resultados de pregunta, hay algo más de polaridad en los hombres. Los encuestados niegan el espacio y lo reconocen como una actividad cotidiana, es decir, que la apropiación del espacio está arraigada, dependiendo de las condiciones cotidianas que corres- ponden a las opciones económicas, lugares de origen, si existen o no familiares o paisanos que los apoyen durante su estadía; sin embargo, estas características dependen de las actividades y oportunidades durante un tiempo y ayuda determinada.

Figura 12. Incidencia del lugar de origen en la creación de las actividades económicas

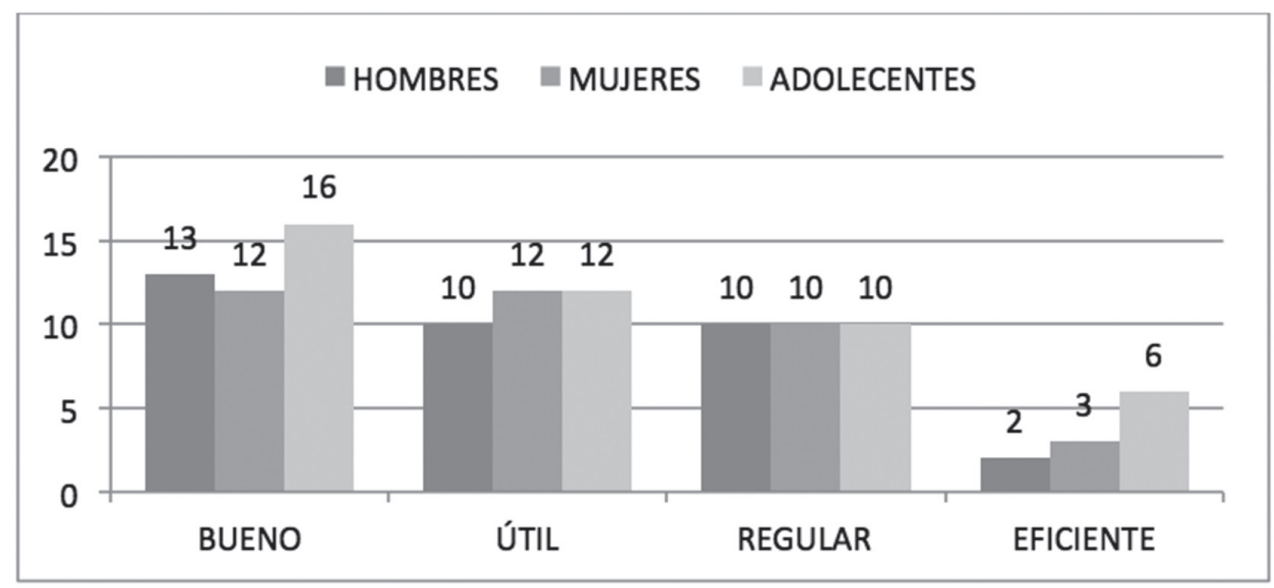

Fuente. Ocampo, Velásquez, 2013.

Aunque la pregunta lleva al punto de "productividad y creación de negocios formales e informales", se evidencia que el $37 \%$ ve con buenos ojos la localización de actividades comerciales dentro del entorno, fortaleciendo su lugar de origen, con la ilusión de traer algo de su cultura, pero tratando de dejar los malos recursos en el pasado y en su origen territorial.
La comunidad termina aceptando y entendiendo que la apropiación del lugar está sujeta a las dinámicas cotidianas y de supervivencia, además de comprender que el espacio público se convierte en el escenario de acercamiento de interacción gracias a la dinámica de desplazamiento, siempre y cuando, puedan articular su destino a las actividades económicas que encuentren.

Figura 13 ¿Quién tiene la responsabilidad de apoyar a la comunidad desplazada por la violencia?

\begin{tabular}{|l|c|c|c|c|}
\hline \multicolumn{3}{c}{ PRIVADO } \\
\cline { 2 - 5 } & \multicolumn{2}{c|}{50} \\
\cline { 2 - 5 } & PRIVADO & PUBLICO & $\begin{array}{c}\text { COMUNITA } \\
\text { RIO }\end{array}$ & ONG \\
\hline M HOMBRES & 13 & 10 & 10 & 2 \\
\hline MUJERES & 12 & 12 & 10 & 3 \\
\hline ADOLECENTES & 16 & 12 & 10 & 6 \\
\hline
\end{tabular}

Fuente. Ocampo, Velásquez, 2013. 
La percepción general indica que la responsabilidad está enmarcada en el sector público, es así que los factores de dignidad, calidad, servicio, precio y destino resultan vitales para el habitante del lugar. Los porcentajes equilibrados indican que la responsabilidad de las políticas públicas urbanas, no satisfacen al sector público, dejando decisiones de desarrollo al privado sin llegar a un acuerdo con todos los actores, pero que en definitiva, se necesita a la población como ingreso activo del desarrollo de los trabajos informales.

Tabla 6. Programas en los últimos gobiernos distritales

\begin{tabular}{|c|c|}
\hline Luis Eduardo Garzón 2004-2008 & $\begin{array}{l}\text { Promoverá atención a la población en condiciones de desplazamiento } \\
\text { forzado, desmovilización o vulnerabilidad frente a la violencia, con una } \\
\text { perspectiva a los Derechos Humanos y al Derecho Internacional Humanitario. } \\
\text { También, promoverá la integración integral. Complementaria a la que } \\
\text { brindan las instituciones del orden nacional, con un enfoque de derechos } \\
\text { humanos, garantizando el cumplimiento del Derecho Internacional } \\
\text { Humanitario. }\end{array}$ \\
\hline Samuel Moreno Rojas 2008-2012 & $\begin{array}{l}\text { Dentro del tema de construcción de paz y reconciliación, Petro pretende } \\
\text { generar condiciones que permitan construir paz, promoviendo la solución } \\
\text { pacífica de conflictos, la no violencia activa, y garantizar efectivamente los } \\
\text { derechos de las víctimas y de la población desplazada, fortaleciendo los } \\
\text { compromisos y acuerdos humanitarios, los pactos ciudadanos de paz, el } \\
\text { aporte a diálogos y negociaciones y la integración de excombatientes sin } \\
\text { discriminación alguna. } \\
\text { Construirá una ciudad en la que se reconozcan las diferencias entre los } \\
\text { distintos sexos -hombres, mujeres-, para que se fortaleza la participación } \\
\text { de niños, niñas, jóvenes y mucho más; la participación de la población } \\
\text { desplazada, para que ellas incidan en la definición, ejecución y seguimiento } \\
\text { de las políticas públicas, ya sea directamente o por medio de los } \\
\text { representantes. } \\
\text { Busca proteger y garantizar la restitución de todos los derechos a las víctimas } \\
\text { de acción de grupos armados y el desplazamiento forzado de niños, niñas } \\
\text { y adolescentes. }\end{array}$ \\
\hline Gustavo Petro 2012-2016 & $\begin{array}{l}\text { Fomentará que los desplazados y víctimas de la violencia, ubicados en zonas } \\
\text { de alto riesgo, accedan a los mecanismos de reparación y retornen a su } \\
\text { lugar de origen si así lo desean. Dinamizar el programa de mejoramiento } \\
\text { de vivienda para la reducción de la vulnerabilidad física en zonas de riesgo } \\
\text { bajo y medio, mientras en las zonas de alto riesgo diversificará las opciones } \\
\text { de vivienda para las familias en reasentamiento y promoverá esquemas } \\
\text { alternativos como el reordenamiento ecoeficiente de barrios y la gestión } \\
\text { participativa del suelo de protección por riesgo, los elementos de la } \\
\text { estructura ecológica principal y el borde urbano rural. }\end{array}$ \\
\hline
\end{tabular}

Fuente. alcaldiabogota.gov.co, 2013. 


\section{CONCLUSIONES}

$\mathbf{L}$ as iniciativas se desarrollan en este campo desde el ámbito de los actores comunitarios, las iniciativas son dimensión general y local en coordinación con los demás gobiernos territoriales.

Crear una junta entre los habitantes del sector, que se encargue de llevar toda esta problemática ante las entidades pertinentes para buscar las mejoras, Además, evaluar las políticas, la eficacia y eficiencia; entender cuáles son las temporalidades con base en las necesidades de la población.

Se recomienda la identificación de las actividades económicas formales e informales, desde la perspectiva de supervivencia de la población urbana, en cuyo caso, los entes territoriales deben adoptar políticas activas de prevención del mismo.
Las tareas o acciones por parte de la política pública deben ser dirigidas a acoger al desplazado en la ciudad, disminuir los efectos inmediatos del desplazamiento y proporcionarle a su familia los mínimos de subsistencia y de servicios sociales

Según la ACNUR, el derecho a la reparación que tienen las víctimas, incluye elementos que deben ser considerados en el diseño de las políticas públicas en aras de cumplir y apoyar a la comunidad que ha sido expulsada de su territorio, teniendo en cuenta que deben tener atención prioritaria la población más vulnerable, los niños, niñas, madres cabezas de familia y adultos mayores.

Los niños y niñas que han sufrido su desplazamiento en la etapa preadolescente, o en la adolescencia, corren riesgos adicionales referentes al ejemplo que puedan recibir en la ciudad, la supervivencia y el no deseo de regresar a su lugar de origen en caso de restituirles las tierras.

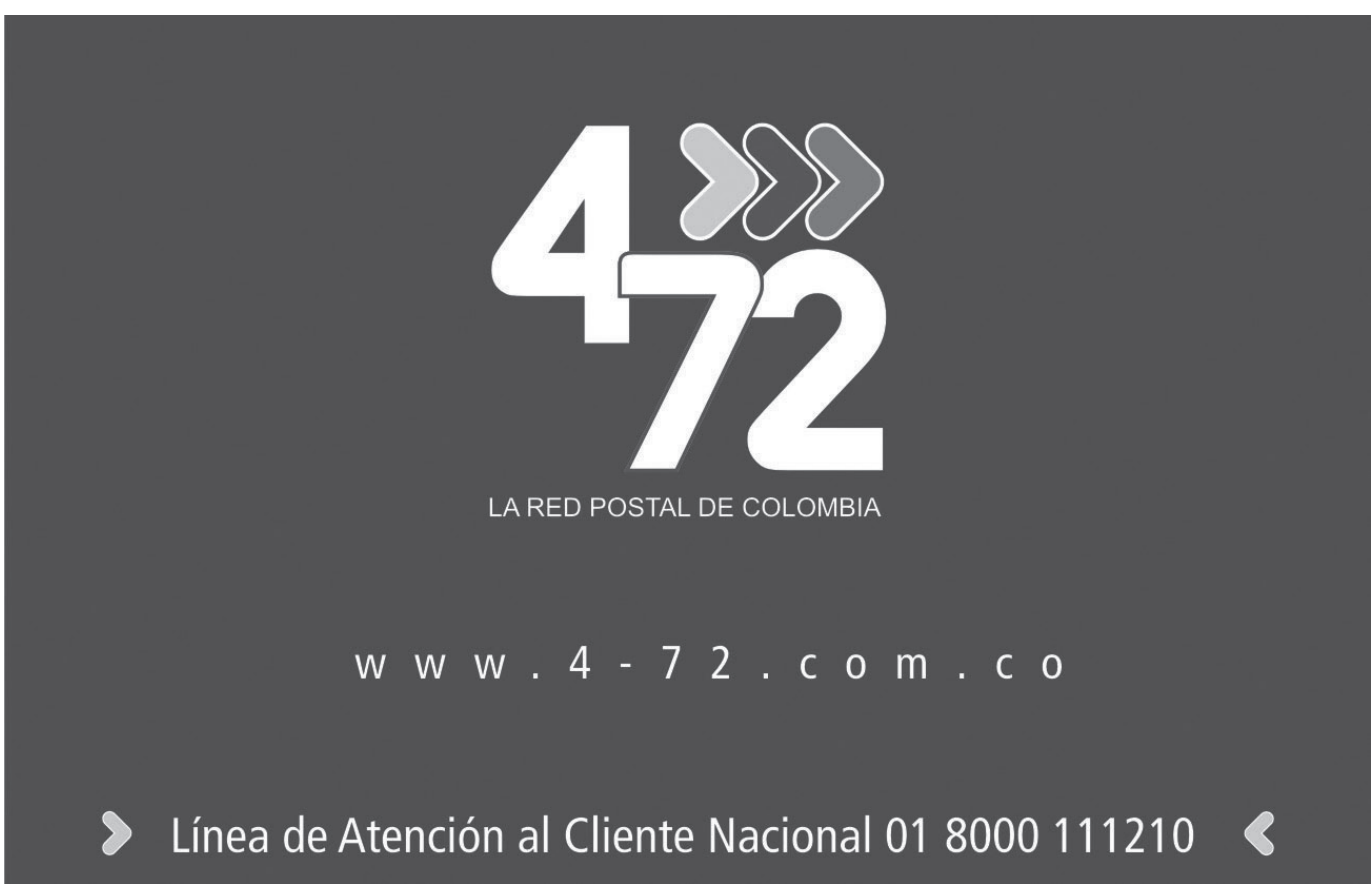




\section{REFERENCIAS BIBLIOGRÁFICAS}

Ceballos, S. A. (2010). Desplazamiento urbano y migración en Colombia. Desplazamiento Urbano, 3.

Distrital, D. A. (2004). El desplazamiento en Bogotá. Una realidad que clama atención. Bogotá: Alcaldía de Bogotá.

Hábitat, S. D. (08 De 07 De 2013). Habitat. vigilancia y control. Bogotá,: Secretaría Distrital Del Hábitat.

Lozano., M. V. (2012). Cuadernos de la ciudad, serie equidad y bienestar. Diagnóstico, Políticas y Acciones en Relación con el desplazamiento forzado hacia Bogotá. En S. D. Distrital, Cuadernos De La Ciudad, Serie Equidad y Bienestar, 4, p. 520). Bogotá: Secretaría De Hacienda Distrital.

Peña, C. R. (2003). Sucesos Migratorios Y Suburbanización. Bitacora, 8.

Schejetnan, M. (1997). Principios de Diseño Urbano/ Ambiental. Col.Sta. Cruz Atoyac: Árbol Editorial S. A De C.V.

Sentencia T-025, T-025 (Corte Constitucional 22 De 02 de 2004).

Alcaldia Bogota. (2005). Recuperado de Http:/Mww.alcaldiabogota.gov.co/Sisjur/Normas/Norma1.Jsp?l=3787\#0:

Alcaldiabogota. (11 De 10 De 2013). Recuperado de: Http:/Mww.Alcaldiabogota.Gov.Co/Sisjur/Normas/Norma1. Jsp?|=3787\#0: Http://www.alcaldiabogota.Gov.Co/Sisjur/Normas/Norma1.Jsp?|=3787\#0

Bogotá Cómo Vamos, R. E. (2012). Recuperado de: Http:/Mww.Bogotacomovamos.Org/Media/Uploads/ Documento/New/Resumen-Ejecutivo-Del-Informe-De-Calidad-De-Vida-En-Bogota-2012.Pdf:

Bogotá, C. D. (2013). Congreso de La República de Colombia. Bogotá: Consejo De Bogotá.

Bogotá, S. D. (2013). Recuperado de: Http:/Www.Habitatbogota.Gov.Co/Sdht/Index.Php?Option=Com_ Content\&View=Article\&Id=644:Secretaria-Del-Habitat-Detecto-16801-Ocupaciones-Ilegales-EnBogota\&Catid=35:Noticias-Principales. 